Measurement of radi al prof i l es of densi ty $r$ at i o of hel i um to hydrogen i on usi ng charge exchange spect roscopy wi th two-wavel engt h spect r omet er

\begin{tabular}{|l|l|}
\hline $\begin{array}{l}\text { j our nal or } \\
\text { publ i cat i on t i t l e }\end{array}$ & Revi ew of Sci ent i f i c I nst r ument s \\
\hline vol une & 86 \\
\hline nunber & 12 \\
\hline page r ange & 123514 \\
\hline year & 2015- 12 - 30 \\
\hline URL & ht t p: //hdl . handl e. net /10655/00012541 \\
\hline
\end{tabular}




\title{
Measurement of radial profiles of density ratio of helium to hydrogen ion using charge exchange spectroscopy with two-wavelength spectrometer
}

\author{
K. Ida, ${ }^{1}$ M. Yoshinuma, ${ }^{1}$ B. Wieland, ${ }^{1}$ M. Goto,${ }^{1}$ Y. Nakamura,${ }^{1}$ M. \\ Kobayashi, ${ }^{1}$ I. Murakami, ${ }^{1}$ C. Moon, ${ }^{1}$ and LHD Experiment Group \\ ${ }^{1}$ National Institute for Fusion Science, Toki, 509-5292, Japan
}

(Dated: November 6, 2015)

\begin{abstract}
Radial profiles of density ratio of helium to hydrogen ions are measured using the charge exchange spectroscopy technique with the two-wavelength spectrometer system in the Large Helical Device. The two-wavelength spectrometer system consists of a dichroic mirror box, a spectrometer with two grating and two camera lenses, and one CCD detector. The dichroic mirror box is used to divide the light of one fiber from the plasma to two fibers, one for HeII ( $\lambda=468.6 \mathrm{~nm})$ and the other for $\mathrm{H}_{\alpha}(\lambda=656.3 \mathrm{~nm})$, that are connected to the entrance slit of the spectrometer to eliminate the interference between the HeII and the $\mathrm{H}_{\alpha}$ spectrum on the CCD. This system provides a simultaneous measurement of helium and hydrogen ion density ratio at 8 exact same locations $(8$ spatial channels) with a time resolution of $>40 \mathrm{~ms}$ in the wide range of the density ratio of $0.05-$ 5 .
\end{abstract}




\section{INTRODUCTION}

Transport of helium is one of the crucial issues in fusion plasma and has been studied by using charge exchange spectroscopy[1-4]. Charge exchange spectroscopy has been widely used as a powerful tool to measure radial profiles of ion temperature and toroidal rotations using the charge exchange lines of impurities[5-7]. Compared with the charge exchange spectroscopy using impurities such as oxygen, carbon, and helium, the cold component emitted from the plasma periphery due to the charge exchange reaction with thermal neutral is relatively large in hydrogen. Recently, the charge exchange spectroscopy was applied to the bulk plasmas by subtracting the emission from the plasma periphery by beam modulation $[8$, 9, 20]. By using beam modulation, radial profiles of helium and hydrogen can be measured with charge exchange spectroscopy.

The isotope mass effect on turbulence and transport is one of the crucial issues in fusion plasma research, and a systematic increase in the amplitude of long-range correlations during the transition from hydrogen- to deuterium-dominated plasmas has been found experimentally[11]. In the Large Helical Device (LHD), the ion cyclotron heating (ICH) experiment in the minority proton heating regime has been performed in the discharges with the mixture of helium and hydrogen instead of the mixture of deuterium and proton, because the heating efficient of ICH is sensitive to the proton fraction[12]. Therefore, the development of the charge exchange spectroscopy system which provides simultaneous measurements of helium and hydrogen density ratio in the core region is recognized to be important. A two-wavelength spectrometer for HeII and HI charge exchange lines was installed in the LHD to derive radial profiles of the density ratio of helium to hydrogen. In this paper, the two-wavelength spectrometer system and the measurements ofdensity ratio of helium to hydrogen are described.

\section{EXPERIMENTAL SETUP FOR HELIUM/HYDROGEN DENSITY RATIO MEASUREMENT WITH CHARGE EXCHANGE SPECTROSCOPY}

Figure 1 shows the experimental setup for the helium/hydrogen density ratio measurement with charge exchange spectroscopy using the two-wavelength spectrometer. The light from the plasma is led to the entrance of the dichroic mirror box and the light with the 
longer wavelength $(\lambda>495 \mathrm{~nm})$ is transmitted through the dichroic mirror, while the light with the shorter wavelength $(\lambda<495 \mathrm{~nm})$ is reflected. By using this dichroic mirror box, the light of one fiber from the plasma is separated into two fibers for helium and hydrogen line measurements and is led to the two-wavelength spectrometer in order to measure the density ratio of helium to hydrogen. The dichroic mirror box consists of one dichroic mirror, two edge filters, three camera lenses, and three fiber holders. The two-wavelength spectrometer (Bunkou-Keiki CLP-400) consists of two $\mathrm{f}=400 \mathrm{~mm} \mathrm{~F}=2.8$ camera lenses and two gratings (1800 l/mm for helium and $1200 \mathrm{l} / \mathrm{mm}$ for hydrogen line) and the CCD detector, as seen in Fig. 1 The dispersion of $1800 \mathrm{l} / \mathrm{mm}$ grating is $1.05 \mathrm{~nm} / \mathrm{mm}$ at HeII line from helium $(\lambda=$ $468.6 \mathrm{~nm})$ and that of $1200 \mathrm{l} / \mathrm{mm}$ is $1.62 \mathrm{~nm} / \mathrm{mm}$ at $\mathrm{H}_{\alpha}$ line from hydrogen $(\lambda=656.3 \mathrm{~nm})$. The two-dimensional toroidal optical fiber array of 50 channels in horizontal and 9 channels in vertical[13] is used for this measurement. The 8 optical fibers from two-wavelength spectrometer system are connected to the two-dimensional toroidal optical fiber array using a connection panel. The location of the measurement can be scanned by changing the connection of fibers at the connection panel.

It is well known that the light ion helium produces a second source of emission from a charged ion that has followed field lines and emits from a volume not localized to where the sightline crosses the beam. This effect is known as plume emission[5, 14]. The plume effect in this measurement is discussed here. The toroidal angle between the viewing port and the neutral beam injection for the charge exchange spectroscopy is 72 degrees. Since the tilt of the magnetic field is relatively large in the edge region in LHD (rotational transform $\iota=0.5$ - 1.2)[15] the vertical separation between the beam center and the sightline at the toroidal angle of the viewing port evaluated by $r_{\text {eff }} / \sqrt{\kappa} \times \sin (72 \times \iota)$ is $0.13-0.51 \mathrm{~m}$, which is $1-4$ times the one-half of the nominal beam height $(\sim 0.12 \mathrm{~m})$. Here, $r_{\text {eff }}$ is the averaged minor radius on a magnetic flux surface and $a_{99}$ is the effective minor radius in which $99 \%$ of the plasma kinetic energy is confined and is $0.63 \mathrm{~m}$, while $\kappa$ is an elongation ratio of poloidal cross section and is 2 . Therefore, the plume effect of $\mathrm{He}^{+}$ions produced at $r_{\text {eff }} / a_{99}>0.5$ can be neglected in this experiment.

The 8 channels of the optical fiber array from the plasma are connected to this system and 16 spectra on the CCD detector give a radial profile of the helium and hydrogen density ratio at 8 locations. The diameter of the core is $200 \mu \mathrm{m}$ and the diameter of the cladding is $250 \mu \mathrm{m}$. The interference between the HeII and the $\mathrm{H}_{\alpha}$ spectra in the CCD is eliminated 
by the dichroic mirror box arranged in front of the two-wavelength spectrometer. The back illuminated electron multiplying $640 \times 512$ pixel $(12 \times 12 \mu \mathrm{m})$ CCD camera (PixelVision PV652) is used as a detector to achieve a high frame rate, $f_{c c d}$, up to $200 \mathrm{~Hz}$ with 21 pixel vertical binning (24 channels). The pitch of the binned strip is $252 \mu \mathrm{m}$, while the pitch of the optical fiber is equal to the outer diameter of the optical fiber used $(250 \mu \mathrm{m})$ if the optical fiber is simply aligned on the entrance slit. Although the displacement between the strip and optical fiber is only $2 \mu \mathrm{m}$ for each fiber, it becomes $48 \mu \mathrm{m}$ for the entire fiber array and causes cross talk between the channels. In order to match the image of the optical fiber into the strip of the CCD $(252 \mu \mathrm{m})$ and minimize the channel cross talk, the optical fiber array is arranged at the slit with a $252 \mu \mathrm{m}$ pitch using the pitch control technique[13].

The amount of the light is adjusted in the wide range of two orders of magnitude by selecting the appropriate electron multiplying (EM) gain of the CCD detector and the F number of the iris of the lens in the dichroic beam splitter box. In general, $\mathrm{H}_{\alpha}$ intensity is stronger than that of HeII intensity. Therefore, the electron multiplying (EM) gain is adjusted to optimize HeII intensity with the iris of the lens for HeII light full open $(\mathrm{F}=1.4)$. Then, the $\mathrm{F}$ number of the iris of the lens for $\mathrm{H}_{\alpha}$ light is selected to be of $\mathrm{F}=4-16$ in order to make the $\mathrm{H}_{\alpha}$ and HeII light to be of similar intensity and to avoid the saturation of the $\mathrm{H}_{\alpha}$ signal on the CCD detector.

Figure 2 shows the transmission of this system using the dichroic mirror and the red/blue edge filter in the two-wavelength spectrometer system. The edge wavelength of the dichroic mirror used in this system is $495 \mathrm{~nm}$, where the light with longer wavelength is transmitted and the light with shorter wavelength is reflected. As seen in Figure 2, there is $6 \%$ transmission in the wavelength region of $\lambda=510-520 \mathrm{~nm}$. This is due to the leaked reflection (reflection of the light above the edge wavelength) from the dichroic mirror. In order to eliminate the interference between the lights from $1200 \mathrm{l} / \mathrm{mm}$ and $1800 \mathrm{l} / \mathrm{mm}$ grating, the additional edge filters of the red side and the blue side are installed in the exit of the dichroic mirror box. This is important for avoiding the overlap of the HeII line $(\lambda=468.6 \mathrm{~nm})$ and the $\mathrm{H}_{\alpha}$ line $(\lambda=656.3 \mathrm{~nm})$. The edge wavelength of the longer wavelength cut-off (blue filter) and shorter wavelength cut-off (red filter) is $534 \mathrm{~nm}$ and $554 \mathrm{~nm}$, respectively. The decrease of transmission due to the blue filter is seen at the wavelength of $534 \mathrm{~nm}$.

The spectra of the HeII line and the $\mathrm{H}_{\alpha}$ line near the plasma periphery at $r_{\text {eff }} / a_{99}=$ 0.89 obtained using this system are plotted in Fig. 3. In this paper, the location of $r_{\text {eff }} / a_{99}$ 
$=1$ is defined as plasma boundary, because that location gives good estimation for the plasma boundary[16]. The region for $r_{\text {eff }} / a_{99} \leq 1$ and $r_{\text {eff }} / a_{99} \geq 1$ are defined as core and scrape-off-layer (SOL), respectively.

The spectrum of the charge exchange line is derived from the difference between the spectrum with neutral beam-on $(t=3.96 \mathrm{~s})$ and neutral beam-off $(\mathrm{t}=4.01 \mathrm{~s})$. As seen

in Fig. 3(a), the emission from plasma periphery is comparable to that due to charge exchange reaction in HeII line, while the charge exchange line from $\mathrm{H}_{\alpha}$ is less than $15 \%$ of the periphery emission as seen in Fig.3(b). Fig.3(c) shows the expanded view of $\mathrm{H}_{\alpha}$ spectra after subtracting the periphery emission with beam on/off modulation. The beam excitation component can not be eliminated by the beam modulation. However, this component has blue shift of few $\mathrm{nm}$, which is large enough to be separated from the charge exchange component.

The intensities of HeII and $\mathrm{H}_{\alpha}$ are calculated from the spectra of charge exchange lines measured using charge exchange spectroscopy (CXS). The fraction of charge exchange line intensity decrease in the core region because of the beam attenuation. The charge exchange fraction becomes too small to drive ion temperature and plasma rotation near the plasma center. Because the charge exchange fraction of $\mathrm{H}_{\alpha}$ line is typically from a few $\%$ to $10 \%$ of emission from plasma periphery, the light intensity should be adjusted to the moderate level (not too small but not saturated) using the iris in the dichroic mirror box.

\section{CALIBRATION OF DENSITY RATIO OF HELIUM TO HYDROGEN}

The method of relative calibration of the density ratio of helium to hydrogen is described in this section. In principle, the density ratio of helium to hydrogen can be obtained by sensitive calibration at the HeII and $\mathrm{H}_{\alpha}$ using the emission charge exchange cross section for $\mathrm{H}^{0}$ (beam $)+\mathrm{H}^{+}$and $\mathrm{H}^{0}$ (beam $)+\mathrm{He}^{2+}$. However, the accuracy of the emission charge exchange cross section for these charge exchange reactions is not high enough to determine the precise density ratio of the helium to hydrogen. Therefore, we calibrate the density ratio obtained in this system with the line-averaged ion densities for hydrogen and helium determined using passive lines of HeII $(\lambda=656.0 \mathrm{~nm})$ and $\mathrm{H}_{\alpha}(\lambda=656.3 \mathrm{~nm})$ in the recombining phase at the termination of the plasma, where the temperature decreases rapidly[17, 18]. In this case the assumption of helium transport is not necessary to evaluate the total ion ratio 
of helium to hydrogen, because all the ions in the plasma experience a recombining phase during the termination period.

Figure 4 shows the time evolution of the electron temperature, the electron density, the charge exchange lines of $\mathrm{H}_{\alpha}$ and HeII, and the passive lines of HeII $(\lambda=656.0 \mathrm{~nm})$ and $\mathrm{H}_{\alpha}(\lambda$ $=656.3 \mathrm{~nm})$. In order to eliminate the uncertainty due to the time and the radial variation, we select the discharge where the density ratio of helium to hydrogen is relatively constant in space and in time until the termination of the plasma as seen in Fig.4. The NBI \#1, \#2, and \#3 are tangential neutral beams with the beam energy of $150-180 \mathrm{keV}$, while the NBI \#4 and \#5 are perpendicular neutral beams with the beam energy of $40 \mathrm{keV}$. The NBI \#4 is injected as a probe beam for charge exchange spectroscopy and is modulated with a frequency of $5 \mathrm{~Hz}$ to subtract the cold component (the emission from plasma periphery). In order to avoid the change of NBI power, the NBI \#5 is injected with the modulation of $5 \mathrm{~Hz}$ in out of phase.

The intensity of the charge exchange lines of $\mathrm{H}_{\alpha}(\lambda=656.3 \mathrm{~nm})$ and HeII $(\lambda=468.6 \mathrm{~nm})$ at $r_{\text {eff }} / a_{99}=0.89$ are constant during the steady-state phase $(\mathrm{t}=4.2-7.0 \mathrm{sec})$, where the central electron density and electron temperature are $2.4 \times 10^{19} \mathrm{~m}^{-3}$ and $3 \mathrm{keV}$, respectively. After the turning-off of the NBI at $\mathrm{t}=7.3 \mathrm{sec}$, the electron temperatures start to decrease in the time scale of $0.3 \mathrm{sec}$, while the electron densities increase slightly. During the decay phase of the electron temperature, significant increases of passive lines of $\mathrm{H}_{\alpha}(\lambda=656.3 \mathrm{~nm})$ and HeII $(\lambda=656.0 \mathrm{~nm})$ are observed. The ion density ratio of helium to hydrogen is evaluated from this intensity ratio in the recombining phase and it is applied to the calibration of helium to hydrogen density ratio measured with charge exchange spectroscopy in the steady-state phase.

In general, the density ratio of helium to hydrogen is not equal to the influx ratio of helium and hydrogen, because of the difference in transport. For example, larger inward convection velocity or smaller diffusion coefficient contribute to the increase of density even with small influx. Therefore, the estimate of density ratio from the influx ratio is quite sensitive to the difference in transport between helium and hydrogen. In contrast, the intensity ratio of helium to hydrogen during the recombining phase does not depend on the transport. This is because all ions are recombined and emit light during the recombining phase as the electron temperature drops after the heating is turned off, regardless of the transport.

The calibration of the density ratio of helium to hydrogen using recombination phase 
has a significant advantage compared with the conventional approach for calculating the density of hydrogen and helium using the beam density calculated and charge exchange cross section in the device where the reflection minor is installed inside the vacuum such as in LHD. Because the reflection rate of the mirror inside the vacuum vessel has a significant wavelength dependence when the mirror is coated by plasma spattering, the sensitivities both for the HeII line $(\lambda=468.6 \mathrm{~nm})$ and the $\mathrm{H}_{\alpha}$ line $(\lambda=656.3 \mathrm{~nm})$ vary in time differently. The reflection for the light with shorter wavelength tends to decrease faster than that of the light with longer wavelength, which makes the evaluation of Helium to hydrogen density ratio difficult. In contrast, the wavelength of the passive lines of HeII $(\lambda=656.0 \mathrm{~nm})$ and $\mathrm{H}_{\alpha}(\lambda=656.3 \mathrm{~nm})$ used for the calibration during the recombination phase is very close and is not influenced by the change in reflection of the mirror due to plasma spattering.

Figure 5 shows the radial profiles of the electron temperature, electron density, beam density, the ratio of the halo component to the beam component, the charge exchange line intensity of $\mathrm{H}_{\alpha}$ and HeII, the hydrogen and helium density, and the density ratio of helium to hydrogen. The radial profiles of beam density are calculated with beam attenuation code based on the density and temperature profiles measured. The peak of the $\mathrm{H}_{\alpha}$ intensity locates further outward than that of HeII at $r_{\text {eff }} / a_{99}=0.95$. The decrease of intensity of HeII in the core $\left(r_{\text {eff }} / a_{99} \leq 1\right)$ toward the plasma center is due to the beam attenuation. The radial profiles of helium and hydrogen density calculated from the HeII and $\mathrm{H}_{\alpha}$ and beam density are also plotted. The hydrogen profiles are slightly peaked, while the helium profiles are flat. The charge exchange spectroscopy system is calibrated to match the averaged helium hydrogen ratio in the core during the steady state phase in the discharge to that evaluated from the HeII and $\mathrm{H}_{\alpha}$ intensity ratio during the recombining phase, as indicated in Fig. 4(e).

It is also well known that the hydrogen produce a second source of emission, which is known as halo emission. Halo emission originates from hydrogenic ions that spatially diffuse away from the beam ballistically and emit photons $[19,20]$. The ratio of halo neutrals to the injected NBI is estimated by using the EIRENE-3D Monte Carlo code[21]. As seen in Fig.5(d), the halo ratio is relatively flat in space and the effect of halo emission to the radial profile of hydrogen is small. Since the ratio of helium density to hydrogen density measured with charge exchange spectroscopy is calibrated using the helium to hydrogen ration in the recombination phase, the halo emission can be neglected in this technique if the ratio is 
unchanged. The halo ratio increases as the plasma density is increased. Therefore, when this measurement is applied to the plasma in which the plasma density is different from the discharge for the calibration, correction due to halo emission is necessary. This issue is treated in the discussion section.

The decrease of $n_{\mathrm{He}} / n_{\mathrm{H}}$ value in the $\mathrm{SOL}\left(r_{\mathrm{eff}} / a_{99} \geq 1\right)$ is not due to the low temperature. The electron density and temperature are high enough to make the helium become fully ionized. The electron density and temperature measured with YAG Thomson scattering at the plasma boundary is $2 \times 10^{19} \mathrm{~m}^{-3}$ and $0.3 \mathrm{keV}$. Therefore, the significant drop of $n_{\mathrm{He}} / n_{\mathrm{H}}$ ratio at the plasma boundary $\left(r_{\text {eff }} / a_{99}=1.0\right)$ is an interesting observation, In this experiment, the $n_{\mathrm{He}} / n_{\mathrm{H}}$ ratio is measured at the mid plane where the helical X-point and stochastic layer located in the SOL.

\section{TIME EVOLUTIONS OF RADIAL PROFILES OF DENSITY RATIO OF HE- LIUM TO HYDROGEN}

Figure 6 shows the radial profiles and time evolution of the density ratio of helium to hydrogen in the discharge with helium puff for $\mathrm{t}=3.4-3.8 \mathrm{sec}$. The line averaged electron density increases from $2 \times 10^{19} \mathrm{~m}^{-3}$ to $5 \times 10^{19} \mathrm{~m}^{-3}$ after the He gas puff. The HeII intensity increases after the onset of the He gas puff and reaches to the steady-state after $t=3.8$ sec. The radial profile of HeII intensity shows a sharp increase at the plasma boundary and the peak of the HeII intensity locates at $r_{\text {eff }} / a_{99}=0.9$. In contrast, the $\mathrm{H}_{\alpha}$ intensity decreases after the onset of the He gas puff because of the increase of beam attenuation due to the density rise. The density ratio of helium to hydrogen increases after the He gas puff and reaches to 2.0. It should be noted that the $n_{\mathrm{He}} / n_{\mathrm{H}}$ value in the SOL stays at the low level of 0.3 .

This result shows that the helium density in the SOL (X-point region) does not increase even if helium gas is puffed. The time evolution of $n_{\mathrm{He}} / n_{\mathrm{H}}$ and the ratio of influx evaluated from $\mathrm{H}_{\alpha}(656.3 \mathrm{~nm})$ and HeI (singlet 2p-3d 667.8nm) are plotted in Fig. 6(b). The He gas puff is applied at $\mathrm{t}=3.4 \mathrm{sec}$ and the gas flow exceeds $10 \mathrm{~Pa} \mathrm{~m} / \mathrm{s}$ and decreases to zero at $=3.8$ sec. Because the recycling rate of helium is higher than that of hydrogen, the $n_{\mathrm{He}} / n_{\mathrm{H}}$ value continues to increase after the turning-off of the He gas puff and reaches the maximum value of 2.0 at $\mathrm{t}=4.1 \mathrm{sec}$. The $n_{\mathrm{He}} / n_{\mathrm{H}}$ value in the core is higher than the influx 
ratio, while that in the SOL is lower than the influx ratio. This result clearly shows that the density ratio in the plasma is not identical to the influx ratio because of the difference in penetration and transport between helium and hydrogen. There is no modification of pumping to enhance the helium pumping efficiency. Therefore, the recycling of helium is close to unity and therefore the decay of helium density after the short pulse of helium puff is relatively long.

As seen in Fig.7(a), the density ratio $f_{\mathrm{He}}\left(=n_{\mathrm{He}} / n_{\mathrm{H}}\right)$ can be controlled by the amount of the He gas puff. Even without the gas puff, the density ratio of $n_{\mathrm{He}} / n_{\mathrm{H}}$ increases in time by the recycling and reaches 0.2 in $t=7 \mathrm{~s}$. When the strong He gas puff is applied early in the discharge at $\mathrm{t}=3.4 \mathrm{~s}$, the density ratio of $n_{\mathrm{He}} / n_{\mathrm{H}}$ increases up to 5 and decreases in time after the turning-on of the He gas puff. The density ratio at $r_{\text {eff }} / a_{99}=0.89$ is slightly higher than the He to Hydrogen ratio of influx. In order to confirm the validity of the $\mathrm{He} / \mathrm{H}$ ratio with charge exchange spectroscopy, the electron density is estimated from $\left(2 \times n_{\mathrm{He}}+n_{\mathrm{H}}\right)$ and compared with the electron density measured with YAG Thomson scattering. As seen in Fig.7(b), there is reasonable agreement between the electron density evaluated from helium and hydrogen ion and electron density in the wide range of $\mathrm{He} / \mathrm{H}$ ratio. The comparison of the electron density evaluated from different diagnostics (bulk charge exchange spectroscopy and YAG Thomson scattering) would be a reliable test of the validity of the calibration method used in this experiment.

\section{DISCUSSION}

The advantage of the two-wavelength spectrometer for the density ratio of helium to hydrogen is discussed. Since the light from one optical fiber is divided into two fibers (one for HeII and the other for $\mathrm{H}_{\alpha}$ ), the location of the measurements are identical for helium and hydrogen. The spectra of HeII and $\mathrm{H}_{\alpha}$ are measured with one CCD detector where the sensitivity for the helium line and the hydrogen line is identical regardless of the EM gain.

In order to obtain the radial profile of the density ratio, the charge exchange spectroscopy technique is adopted. In general, the absolute value of the charge exchange cross section has some uncertainty which is too large for determining the precise density ratio of helium to hydrogen. In order to calculate the ion density, the beam density (beam attenuation)

should be calculated based on the temperature and the electron and ion densities of each 
species. However, the ion density for each species is unknown and therefore the iteration process is required to calculate the beam density. In the system described in this paper, the calculation of beam density is necessary to derive the ion density profile, but it is not necessary for the density ratio of helium to hydrogen, because the beam attenuation is identical for HeII and $\mathrm{H}_{\alpha}$ measurements. This is an important advantage especially at the higher density where beam density becomes more sensitive to the density of each species due to larger attenuations.

It should be noted that this system does not require the calculations of beam attenuation and halo emission, which have relatively large uncertainty for deriving the radial profile of helium to hydrogen ratio. However, in order to calculate the ratio of helium to hydrogen more accurately, the systematic error due to the lack of the calculations of beam attenuation and halo emission is discussed. The correction factor due to the difference in charge exchange cross section between helium and hydrogen and the change in the halo ratio due to the variation of plasma density is evaluated. Figure 8(a) and (b) show the radial profile of the correction factor for the ration of helium to hydrogen due to the cross section effect and the halo effect. As the electron density is increased, the beam component with one-third and one-half energy (E/3 and E/2) attenuates more than the primary energy (E). Because the energy dependence of charge exchange cross section is different between helium and hydrogen, the ratio of helium to hydrogen is overestimated as the density is increased. In contrast, because the halo ratio increases as the density is increased, the ratio of helium to hydrogen is underestimated as the density is increased by ignoring halo emission. The systematic error due to these effects are $10 \sim 20 \%$ of the ratio of helium to hydrogen and are typically smaller than the statistic error bar of the measurements.

In conclusion, the radial profiles of the density ratio of helium to hydrogen are measured with the two-wavelength spectrometer installed in LHD. The density ratio of helium to hydrogen is found to be not constant in space and to decrease sharply at the plasma boundary even with the He gas puff. The radial profile of the density ratio of helium to hydrogen becomes peaked as the fraction of helium decreases. This result suggests that the impurity (minority species) tends to be more peaked than the bulk ions (majority species).

We would like to thank the technical staff of LHD for their effort to support the experiment in LHD. This work is partly supported by a Grant-in-Aid for Specially-Promoted Research (No. 21224014) and a Grant-in-Aid for Scientific Research (No. 23246164) of JSPS 
Japan. This work is also partly supported by the National Institute for Fusion Science grant administrative budget, NIFS10ULHH021.

[1] R.J. Fonck and R. Hulse, M. Finkenthal, Phys. Rev. Lett. 52 (1984) 530.

[2] D.F. Finkenthal, The Measurement of Absolute Helium Ion Density Profiles on the DIII-D Tokamak Using Charge Exchange Recombination Spectroscopy, PhD. Thesis, University of California, Berkeley (1994).

[3] A. Kappatou, Investigations of helium transport in ASDEX Upgrade plasmas with charge exchange recombination spectroscopy, PhD. Thesis, Eindhoven University of Technology (2014).

[4] K.T.Y. Liao, Helium charge exchange recombination spectroscopy on Alcator C-Mod Tokamak, PhD. Thesis, The University of Texas at Austin (2014).

[5] R.J. Fonck, D.S.Darrow, and K.P.Jaehnig, Phys. Rev. A 29 (1984) 3288.

[6] K. Ida and S.Hidekuma, Rev Sci Instrum. 60 (1989) 867.

[7] K. Ida, S. Kado, and Y. Liang, Rev Sci Instrum. 71 (2000) 2360.

[8] E. Busche, H. Euringer, and R. Jaspers, Plasma Phys. Control. Fusion 39 (1997) 1327.

[9] T. Pütterich, E. Wolfrum, R. Dux, and C. Maggi, Phys. Rev. Lett 102 (2009) 025001.

[10] B. A. Grierson, K. H. Burrell, C. Chrystal, R. J. Groebner, D. H. Kaplan, W. W. Heidbrink, J. M. Muñoz Burgos, N. A. Pablant, W. M. Solomon, and M. A. Van Zeeland, Rev. Sci. Instrum. 83 (2012) 10D529.

[11] Y. Xu, C. Hidalgo, I. Shesterikov, A. Krämer-Flecken, S. Zoletnik, M. Van Schoor, M. Vergote, and the TEXTOR Team, Phys. Rev. Lett. 110 (2013) 265005.

[12] H. Kasahara, T. Seki, K. Saito, R. Seki, R. Kumazawa, Y. Yoshimura, S. Kubo, T. Shimozuma, H. Igami, H. Takahashi, K. Nagasaki, Y. Ueda, M. Tokitani, N. Ashikawa, M. Shoji, T. Wakatsuki, S. Kamio, H. Tsuchiya, S. Yoshimura, N. Tamura, C. Suzuki, H. Yamada, T. Mutoh and LHD Experiment Group Phys. Plasmas 21 (2014) 061505.

[13] M. Yoshinuma, K.Ida, M.Yokoyama, M.Osakabe, K.Nagaoka, Fusion Sci. Technol. 58 (2010) 375 .

[14] R.E. Bell , Rev Sci Instrum. 77 (2006) $10 \mathrm{E} 902$.

[15] K. Ida, S. Kado, and Y. Liang, Rev Sci Instrum. 76 (2005) 053505.

[16] K. Kamiya, K. Ida, M. Yoshinuma, C. Suzuki, Y. Suzuki, M. Yokoyama, Nucl. Fusion 53 
(2013) 013003.

[17] M. Goto, K. Sawada, and T. Fujimoto, Phys. Plasmas 9 (2002) 4316.

[18] M. Goto, S.Morita, K.Sawada, T.Fujimoto, S.Yamamoto, J.Miyazawa, H.Yamada, K.Toi, Phys. Plasmas 10 (2003) 1402.

[19] J.T. Hogan, J. Nucl. Materials 111\&112 (1982) 413.

[20] B.A. Grierson, et. al., Rev. Sci. Instrum. 83 (2012) $10 D 529$.

[21] D. Reiter et al., Fusion Sci. Technol. 47 (2005) 172. 
Figure captions

Fig.1 Experimental setup for helium/hydrogen density ratio measurement with charge exchange spectroscopy using two-wavelength spectrometer.

Fig.2 Transmission of HeII channel and $\mathrm{H}_{\alpha}$ channel through the dichroic mirror plus red and blue filters in the two-wavelength spectrometer system.

Fig.3 Spectrum of (a) HeII, (b) $\mathrm{H}_{\alpha}$, and (c) $\mathrm{H}_{\alpha}$ charge exchange and beam excitation lines measured with the two-wavelength spectrometer.

Fig.4 Time evolution of (a) NBI power, (b) electron temperature, (c) electron density, (d) charge exchange lines of $\mathrm{H}_{\alpha}(\lambda=656.3 \mathrm{~nm})$ and HeII $(\lambda=468.6 \mathrm{~nm})$ at $r_{\text {eff }} / a_{99}=0.89$, and (e) passive lines of $\mathrm{H}_{\alpha}(\lambda=656.3 \mathrm{~nm})$ and HeII $(\lambda=656.0 \mathrm{~nm})$.

Fig.5 Radial profiles of (a) electron temperature, (b) electron density, (c) beam density, (d) the ratio of halo component to beam component, (e) charge exchange line intensity of $\mathrm{H}_{\alpha}$ and HeII, (f) helium and hydrogen density, and (g) density ratio of helium to hydrogen evaluated from CXS measurement and passive spectroscopy during recombining phase.

Fig.6 (a) Radial profiles and (b) time evolution of density ratio of helium to hydrogen in the discharge with helium puff. The dashed lines are the influx ratio evaluated from $\mathrm{H}_{\alpha}(656.3$ nm) and HeI (singlet 2p-3d 667.8nm).

Fig.7 Time evolution of (a) helium to hydrogen ratios, $f_{\mathrm{He}}\left(=n_{\mathrm{He}} / n_{\mathrm{H}}\right)$, at $r_{\mathrm{eff}} / a_{99}=0.89$ and (b) the electron density estimated from $\left(2 \times n_{\mathrm{He}}+n_{\mathrm{H}}\right)$ (solid circles) and the electron density measured with YAG Thomson scattering (solid lines). The dashed lines in Fig(a) are the influx ratio evaluated from $\mathrm{H}_{\alpha}(656.3 \mathrm{~nm})$ and $\mathrm{HeI}$ (singlet 2p-3d 667.8nm).

Fig.8 Radial profiles of correction factor due to the effect of (a) energy dependence of charge exchange cross section and (b) halo emission for the plasma with various density. 


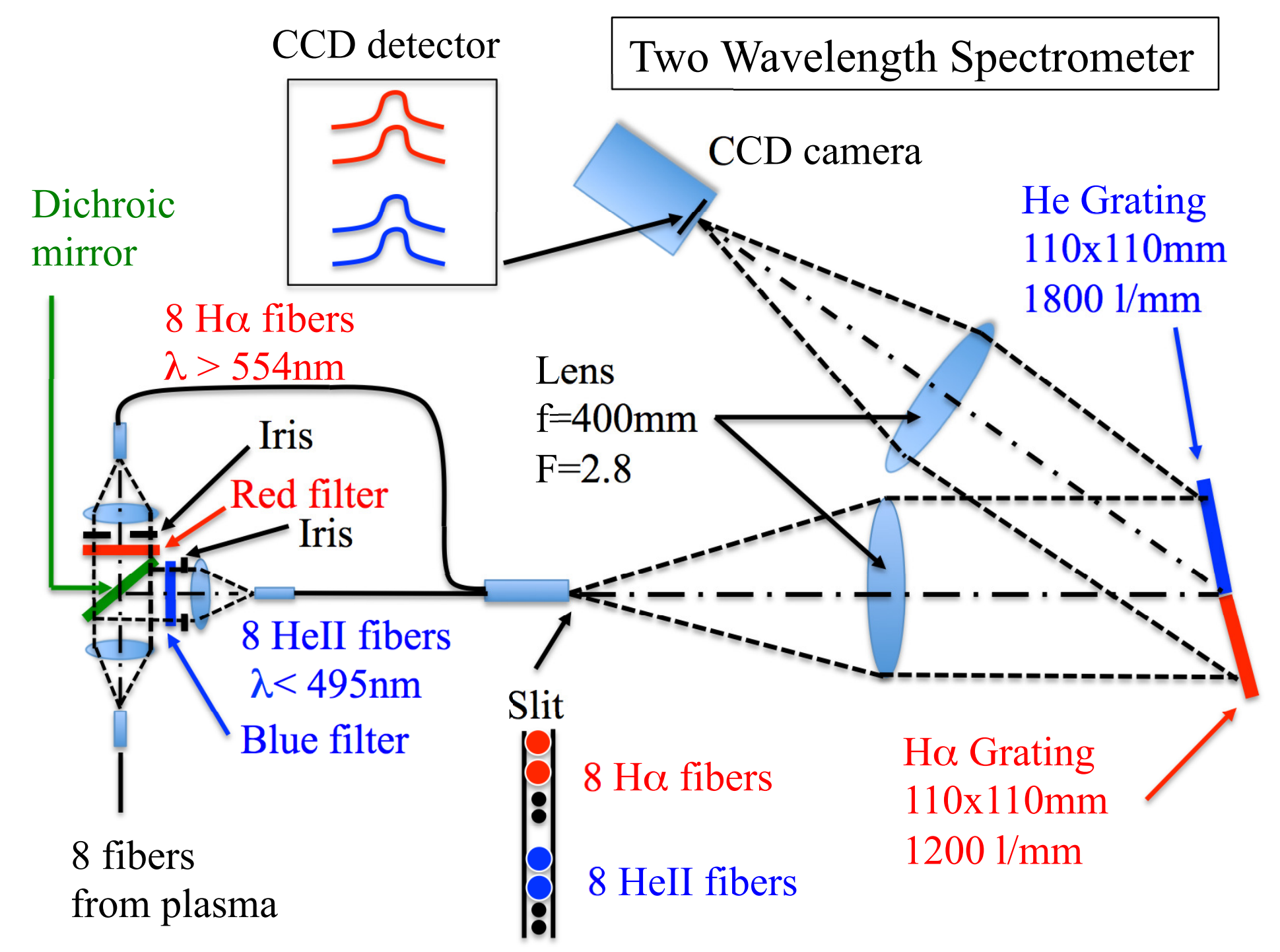




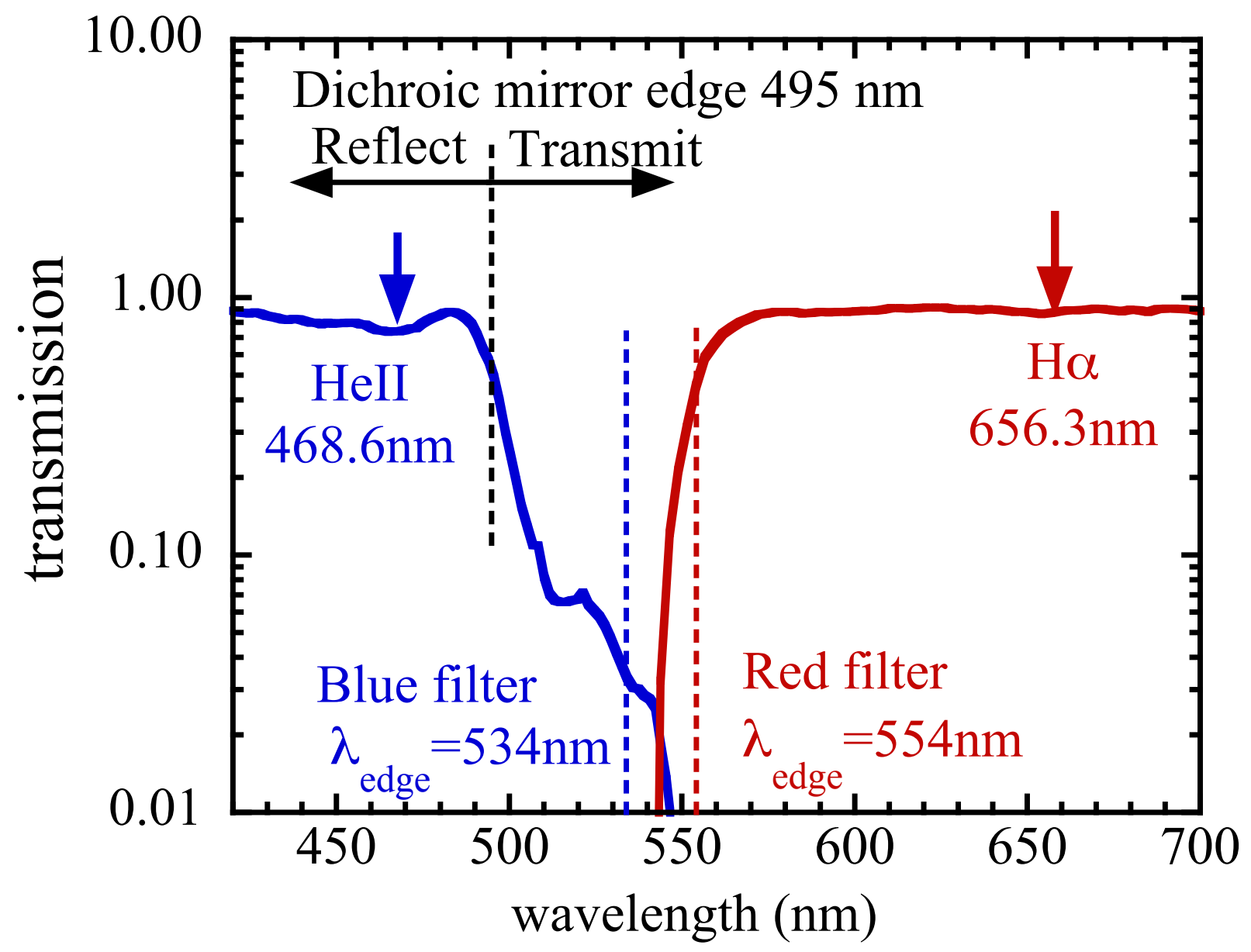



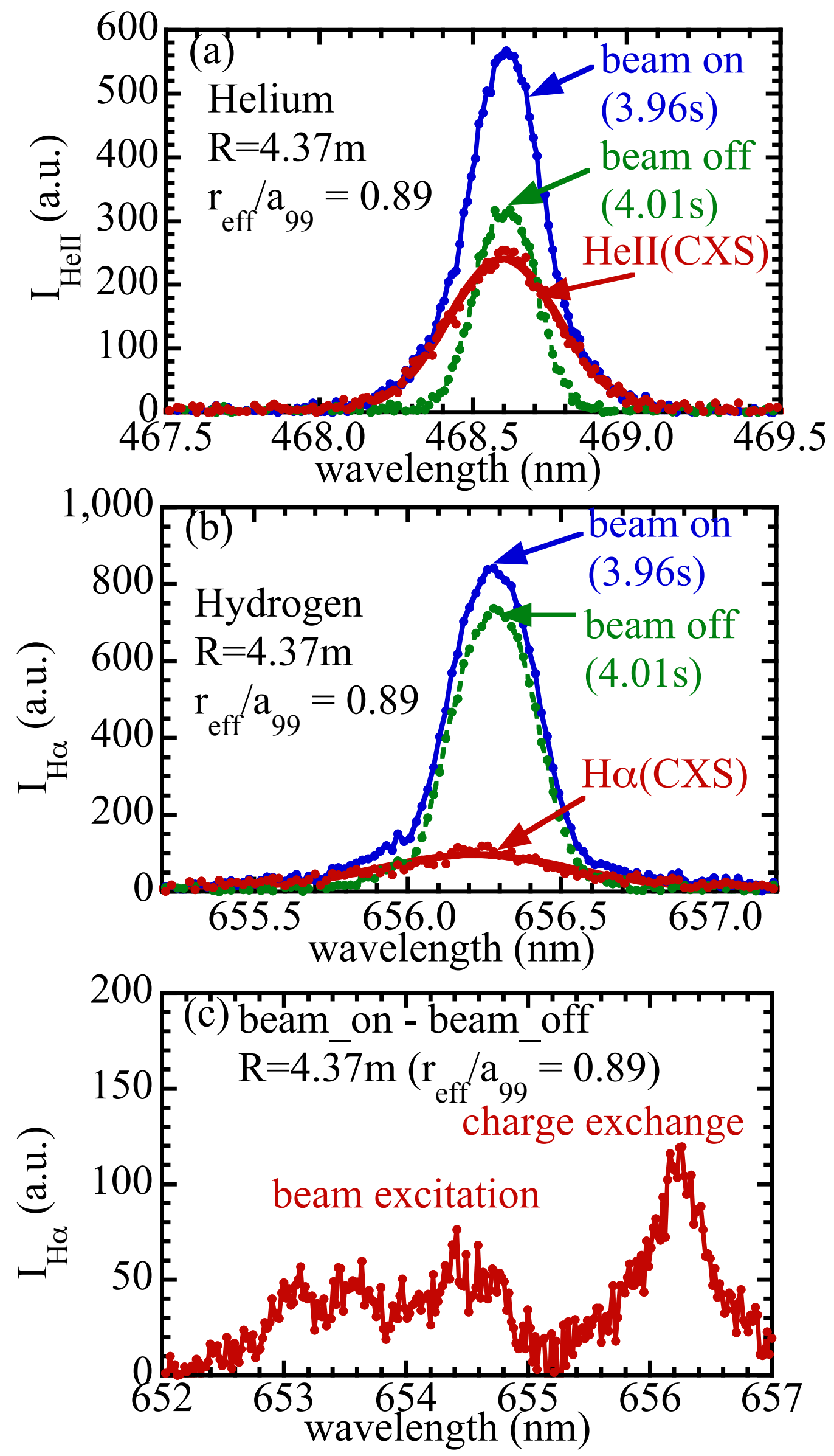


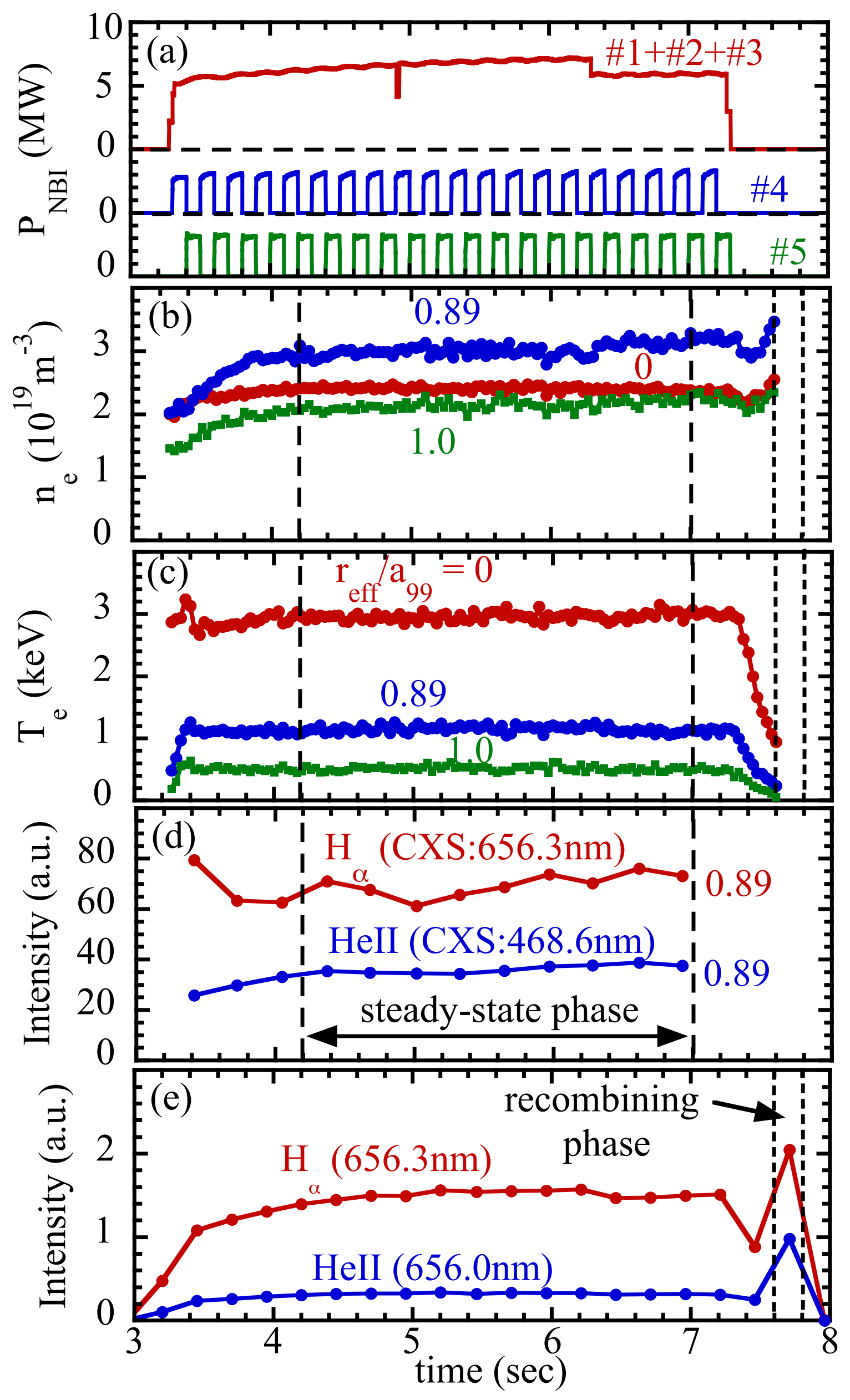




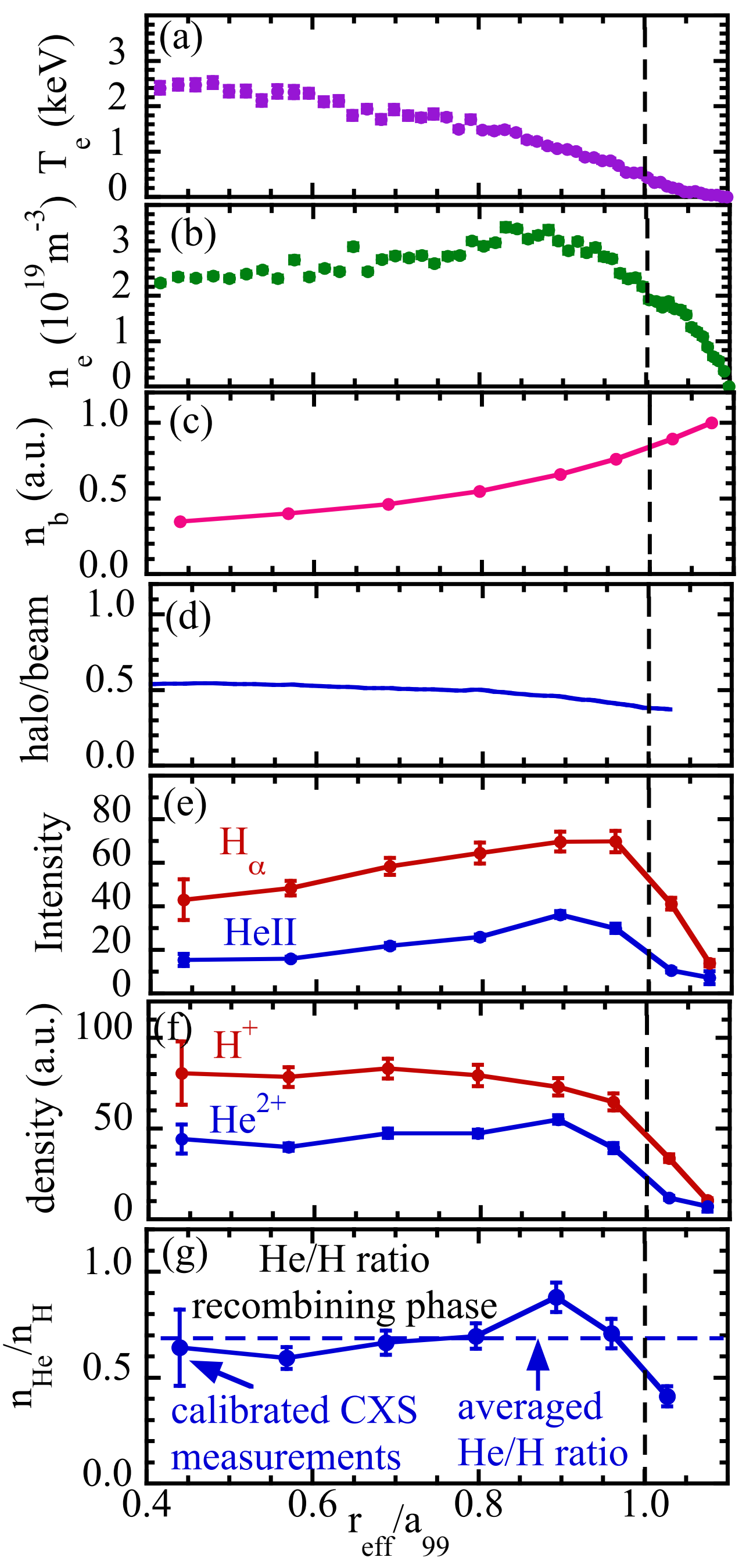



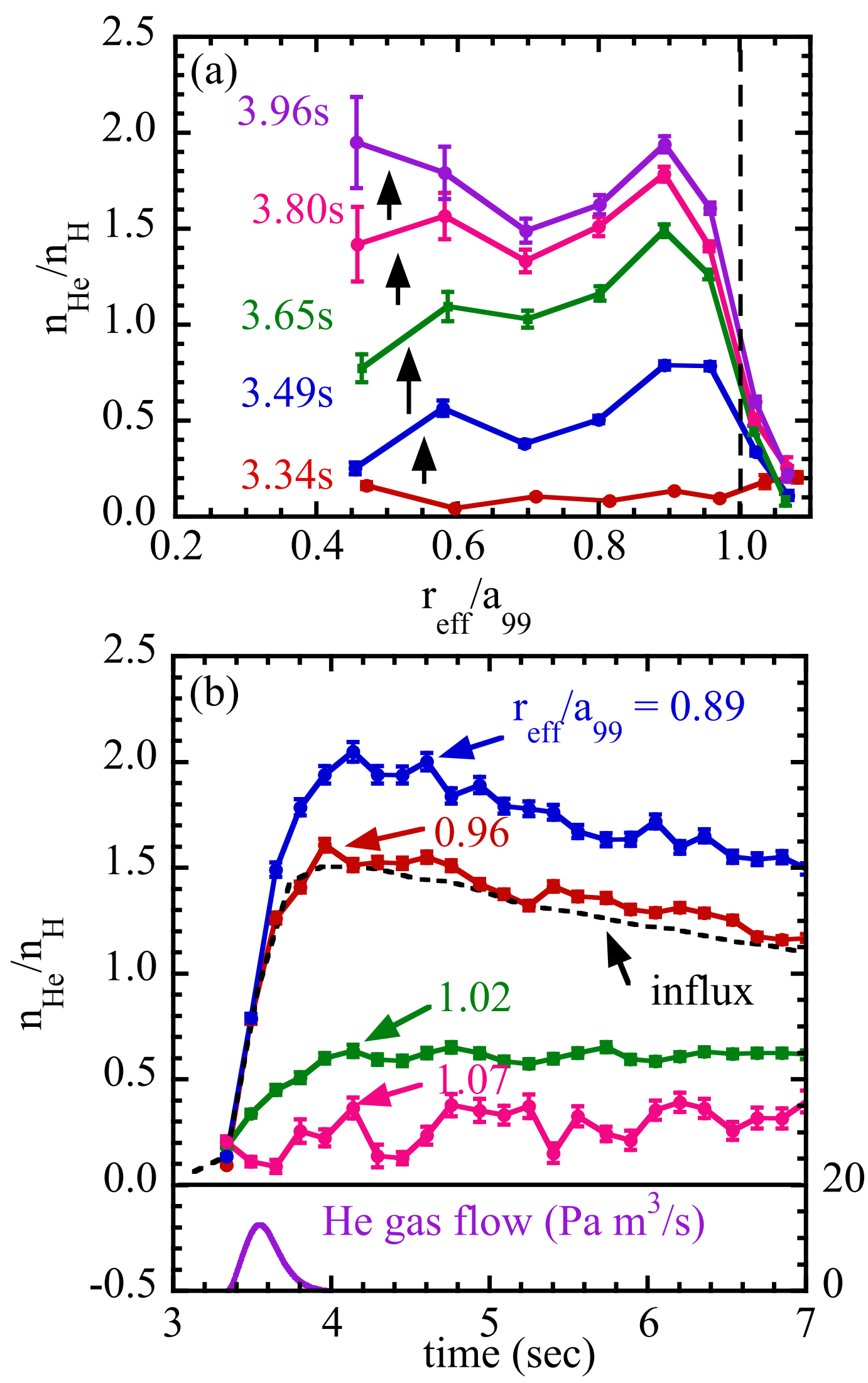


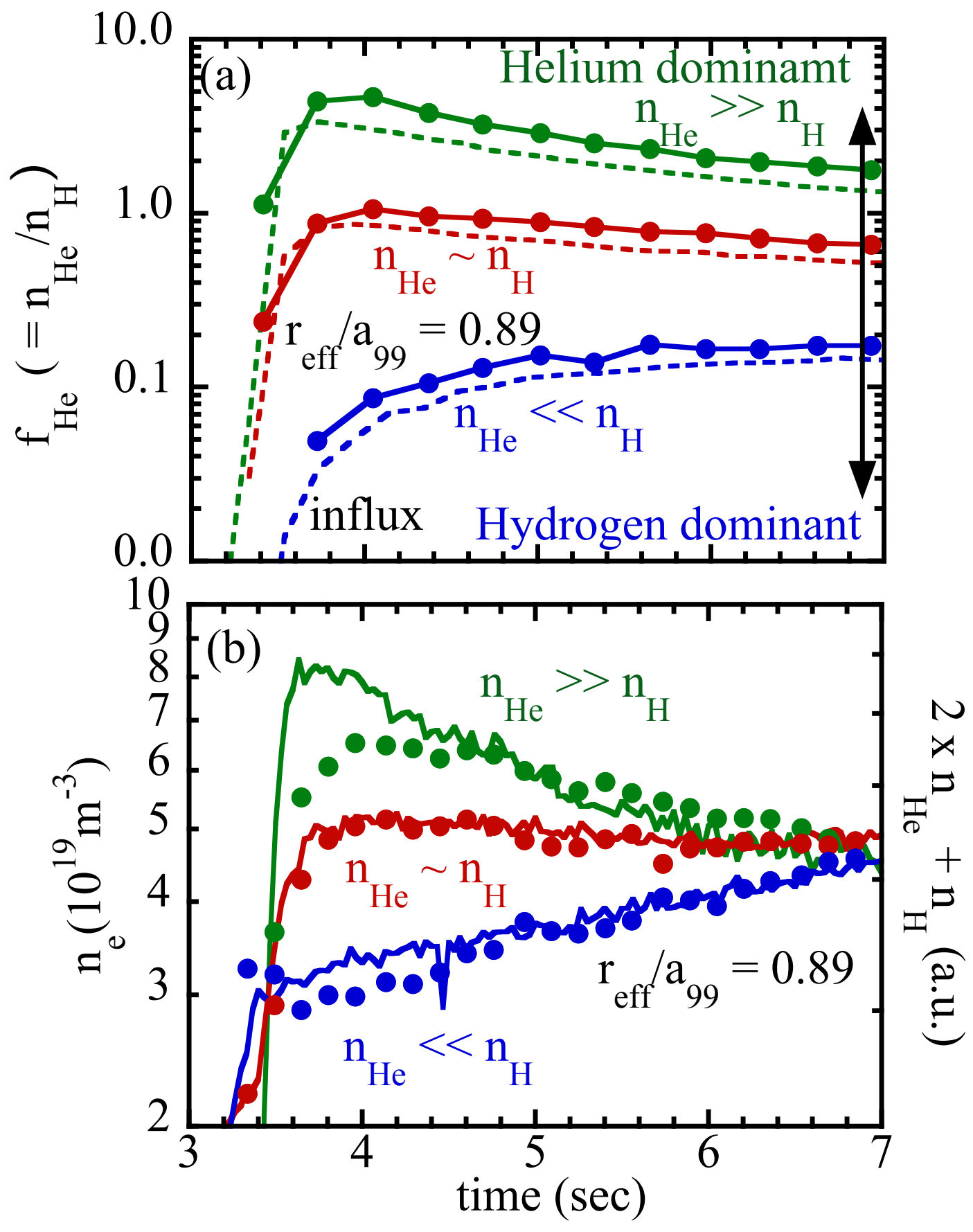




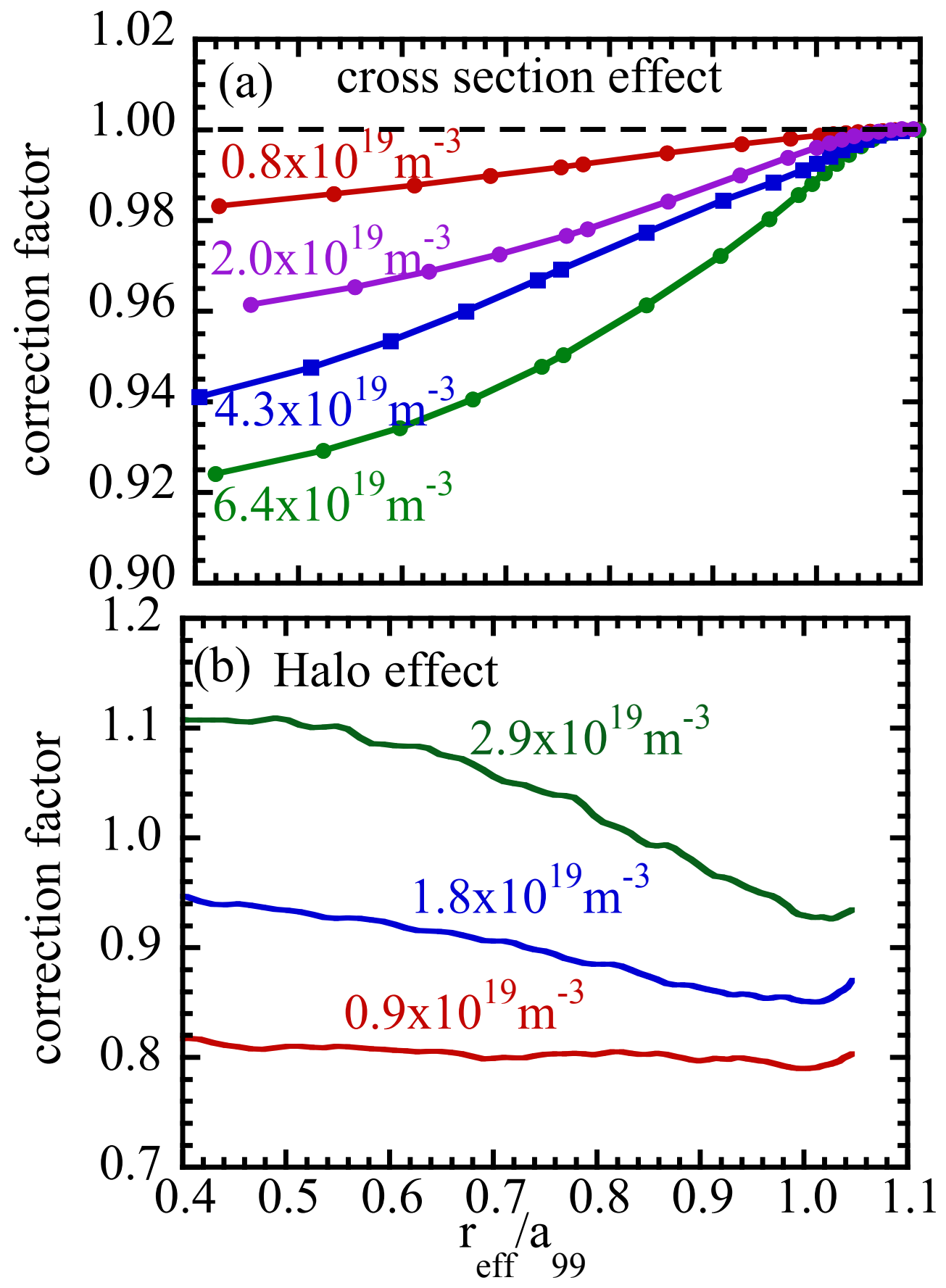

